\title{
Genetic analysis of typical wet-type age-related macular degeneration and polypoidal choroidal vasculopathy in Japanese population
}

\author{
Asako Goto • Masakazu Akahori • Haru Okamoto • Masayoshi Minami • \\ Naoki Terauchi • Yuji Haruhata - Minoru Obazawa • Toru Noda • Miki Honda • \\ Atsushi Mizota • Minoru Tanaka • Takaaki Hayashi • Masaki Tanito • Naoko Ogata • \\ Takeshi Iwata
}

Received: 12 September 2009 / Accepted: 6 November 2009 /Published online: 22 December 2009

(C) The Author(s) 2009. This article is published with open access at Springerlink.com

\begin{abstract}
Age-related macular degeneration (AMD) is a common cause of blindness in the elderly. Caucasian patients are predominantly affected by the dry form of AMD, whereas Japanese patients have predominantly the wet form of AMD and/or polypoidal choroidal vasculopathy (PCV). Although genetic association in the 10q26 (ARMS2/HTRA1) region has been established in many ethnic groups for dry-type AMD, typical wet-type AMD, and $\mathrm{PCV}$, the contribution of the $1 \mathrm{q} 32(\mathrm{CFH})$ region seem to differ among these groups. Here we show a single
\end{abstract}

The first two authors Goto and Akahori have contributed equally to this work.

Electronic supplementary material The online version of this article (doi:10.1007/s12177-009-9047-1) contains supplementary material, which is available to authorized users.

\footnotetext{
A. Goto $\cdot$ M. Akahori $\cdot$ H. Okamoto $\cdot$ M. Minami $\cdot$ M. Obazawa $\cdot$

T. Iwata $(\bowtie)$

Division of Molecular and Cellular Biology,

National Institute of Sensory Organs,

National Hospital Organization Tokyo Medical Center,

2-5-1 Higashigaoka,

Meguro-ku, Tokyo 152-8902, Japan

e-mail: iwatatakeshi@kankakuki.go.jp
}

N. Terauchi $\cdot$ Y. Haruhata $\cdot$ M. Obazawa $\cdot$ T. Noda

Division of Ophthalmology,

National Hospital Organization Tokyo Medical Center,

2-5-1 Higashigaoka,

Meguro-ku, Tokyo 152-8902, Japan

M. Honda $\cdot$ A. Mizota $\cdot$ M. Tanaka

Department of Ophthalmology,

Juntendo University Urayasu Hospital,

2-1-1, Tomioka,

Urayasu 279-0021, Japan nucleotide polymorphism (SNP) in the ARMS2/HTRA1 locus is associated in the whole genome for Japanese typical wet-type AMD (rs10490924: $p=4.1 \times 10^{-14}, \mathrm{OR}=4.16$ ) and PCV (rs10490924: $p=3.7 \times 10^{-8}, \mathrm{OR}=2.72$ ) followed by $C F H$ (rs800292: $p=7.4 \times 10^{-5}, \mathrm{OR}=2.08 ; p=2.6 \times 10^{-4}, \mathrm{OR}=$ 2.00), which differs from previous studies in Caucasian populations. Moreover, a SNP (rs2241394) in complement component $C 3$ gene showed significant association with PCV $\left(p=2.5 \times 10^{-3}, \mathrm{OR}=3.47\right)$. We conclude that dry-type AMD, typical wet-type AMD, and PCV have both common 
and distinct genetic risks that become apparent when comparing Japanese versus Caucasian populations.

Keywords Age-related macular degeneration $\cdot$ Polypoidal choroidal vasculopathy $\cdot$ ARMS2 $\cdot$ HTRA $1 \cdot \mathrm{CFH} \cdot \mathrm{C} 3$

\section{Introduction}

Age-related macular degeneration (AMD) is the most common cause of blindness in elderly people of European descent and affects more than 1.75 million individuals in the USA alone [1]. In Japan, the prevalence of AMD has risen from $0.87 \%$ in 1988 to $1.3 \%$ in 2003 (Japan Ophthalmologists Association Study Group Report 2006-2008). Steady increase of AMD due to longer life span and improvement of diagnostic methods has been observed around the country. The most recent report by Kawasaki et al. describes the prevalence of early stage AMD in the Funagata study (Funagata-cho, Yamagata Prefecture, Japan) as $4.4 \%$ while the prevalence of late stage AMD was $1.1 \%$, showing no difference from a Caucasian study in Australia [2].

AMD is considered a multifactorial disease with involvement of genetic, behavior, and environmental factors, and primarily affects the macular region of the retina [3]. Clinical phenotypes of AMD are manifold. Small and hard drusen often appear in normal-aged eyes and do not necessarily cause AMD [4]. In Caucasians, the early stage of AMD is associated with an increase in the number of large soft drusen and progresses to either the dry form or wet form of the disease [3]. In contrast, Japanese patients predominantly exhibit wet-type AMD with choroidal neovascularization and few or no drusen [5]. Maruko et al. have classified wet-type AMD patients into three subgroups, namely typical wet-type AMD, polypoidal choroidal vasculopathy (PCV), and retinal angiomatous proliferation (RAP) [5]. From 289 Japanese patients examined with wet-type AMD, 35.3\%, 54.7\%, and 4.5\% were diagnosed with typical AMD, PCV, and RAP, respectively. In the remaining 5.5\% patients, one eye had PCV and the other eye had typical AMD. Thus, PCV is the predominant subgroup of wet-type AMD in the Japanese population. $\mathrm{PCV}$ is characterized by branching of the choroidal vasculature basal to the RPE comprising various sized polypoidal structures connected to the branching vascular network, which can be clearly seen by indocyanine green angiography [6]. PCV can be misdiagnosed as typical AMD if only fluorescein angiography is performed. The recent increase in prevalence of PCV is mainly due to the improvement of diagnostic methods. In most Japanese patients, typical wet-type AMD and PCV occur unilaterally (94.1\% and $81.6 \%$, respectively) and show a male preponderance ( $71.6 \%$ and $77.8 \%$, respectively) [5] consis- tent with studies of Eastern Asian patients in China and Korea [7, 8]. However, in Caucasians, PCV predominantly affects women and occurs bilaterally with a prevalence ranging from $4 \%$ to $14 \%$, which is comparably lower than the Eastern Asian populations [6]. On the other hand, it has been reported that the incidence of PCV in black individuals exceeds that of Asians [6]. Although the phenotypic diversity of AMD has been speculated to be associated with differences in genetic background, this has not been clearly established.

Initial efforts to investigate the genetic basis of AMD utilized family studies. A concordance for AMD phenotypes in twins, and a higher risk of siblings of individuals with AMD have been reported [9-14]. These early studies lead to genome-wide linkage analyses using microsatellite markers to search for chromosomal regions associated with affected individuals [15-24]. Several candidate regions including 1q32 and 10q26 were confirmed by a metaanalysis [25]. Progress in genotyping and sequencing technology extended detailed genetic association studies to the entire genome. Age-related eye disease studies (AREDS) of AMD case-control subjects using 100,000 SNPs resulted in the identification of four chromosomal regions significantly associated with the disease, namely CFH (1q32), ARMS2/HTRA1 (10q26), complement component $2 /$ complement factor $\mathrm{B}(C 2 / B F, 6 \mathrm{p} 21)$, and complement component $3(C 3,19 \mathrm{p} 13)$ [26], which is consistent with another genome wide association study recently reported by Swaroop et al. (IOVS 2009; 50:ARVO EAbstract 1614). It should be noted that these genome-wide scans have been conducted on subjects with both the dry and wet form of AMD, with the majority of cases representing the dry form of the disease. However, Zang et al. have identified 34 SNPs which were associated with AMD at $p$ value of less than $10^{-6}$ in the AREDS Caucasian cohort having typical wet-type AMD [27]. They showed that $1 \mathrm{q} 32$ and 10q26 were also significantly associated with typical wet-type AMD. To date, there are no genome-wide genetic studies reported for PCV.

Direct examinations of SNPs in chromosomal regions identified by genome-wide linkage analysis showed that two genomic loci 1q32 and 10q26 including the $C F H$, ARMS2, and HTRA1 genes were associated with AMD in Caucasians and individuals of Hong Kong [28-32]. The association between AMD and three SNPs in these gene regions, namely rs1061170 (CFH), rs10490924 (ARMS2), and rs11200638 (HTRA1), were verified by a number of research groups around the world. We also confirmed the association of rs10490924 and rs11200638 with typical wet-type AMD in the Japanese cohort [33]. From our results and others, rs10490924 and rs11200638 have been shown to strongly associate with dry-type AMD [34], typical wet-type AMD [31, 32, 34], and PCV [35]. It is still unclear how these SNPs contribute to the development of 
different types of AMD. On the other hand, no association was confirmed for rs 1061170 in Japanese AMD [36-40]. This is probably due to the lower allele frequency of $0.07 \pm$ 0.02 for this variant in Eastern Asian population compared to the higher frequency of $0.34 \pm 0.03$ in Caucasians [41]. However, rs800292, another coding SNP in the $C F H$ gene region originally associated with AMD in Caucasians [28], has been shown to associate with typical wet-type AMD and PCV in Japanese and Chinese populations [39, 42-44]. Thus, there is a clear difference in genetic risk for AMD based on ethnicity.

Since the association between the $C F H$ gene and AMD has been established, other components of the complement pathway have been thoroughly examined in Caucasian populations. Among them, the 19p13, 6p21 and 4q25 loci, including the $C 2, B F, C 3$, and complement factor I (CFI) genes, show strong association with AMD [45-49]. However, it still unclear whether $C F I$ or the nearby PLA2G12A gene is associated with the disease. Lee et al. analyzed the same AMD associated SNPs in the C2/BF gene region and reported that there were no differences between Chinese PCV patients and control groups [43]. Similar results were obtained for PCV in Japanese population for SNPs in the $C 2 / B F$ gene region [50]. However, in this study, significant association of diseaseprotective haplotype was observed. There is currently no evidence that the $C 2 / B F$ gene region is a risk for wet-type $\mathrm{AMD}$ or the $C 3$ gene region a risk for typical wet-type AMD and PCV for Eastern Asians. With the exception of 1q32, $10 \mathrm{q} 26,19 \mathrm{p} 13,6 \mathrm{p} 21$, and $4 \mathrm{q} 25$ regions, association of other loci with AMD pathogenesis reported by some genome-wide genetic studies remains unclear [15-24]. These SNP variants may be ethnic specific as is the case for $\mathrm{CFH}$.

To investigate the involvement of genetic factors in Japanese patients who progressed to typical wet-type AMD and PCV, over 500,568 SNPs covering ARMS2/HTRA1, $C F H, C 2 / B F, C 3$, and $C F I$ and other regions were genotyped using Affymetrix Human Mapping $500 \mathrm{~K}$ Arrays and TaqMan assay.

\section{Materials and methods}

\section{Subjects}

One hundred Japanese patients with typical wet-type AMD but without PCV (average age 74.56 \pm 8.83 years), classified as $5 \mathrm{~b}$ by Seddon et al. [51], 100 Japanese patients with PCV (average age, $72.71 \pm 8.25$ years), and 190 agematched Japanese controls (average age, $72.22 \pm 8.51$ years) were recruited for this study (Table 1). All patients were diagnosed by ophthalmoscopic, fluorescein, and indocyanine green angiographic findings. In controls, no signs of
Table 1 Summary of study populations

\begin{tabular}{lllll}
\hline & AMD & PCV & Control & $p$ value \\
\hline No. of subjects & 100 & 100 & 190 & \\
Male/female & $73: 27$ & $81: 19$ & $86: 104$ & $5.0 \times 10^{-10}$ \\
Mean \pm SD & $74.56 \pm 8.83$ & $72.71 \pm 8.25$ & $72.22 \pm 8.51$ & 0.08 \\
Range & $51-90$ & $52-89$ & $50-89$ & \\
\hline
\end{tabular}

$p$ values were obtained by Pearson's chi-square test $\left(5.0 \times 10^{-10}\right)$ and one-way ANOVA (0.08) among three groups

$A M D$ typical wet-type AMD

early AMD, such as soft drusen or alterations of the retinal pigment epithelium in the macula area, were observed ophthalmoscopically. Informed consent was obtained from all participants and the procedures used conformed to the tenets of the Declaration of Helsinki.

\section{Genotyping}

DNA was extracted from blood samples using the Magtration System 8Lx (Precision System Science Co., Ltd., Tokyo, Japan), and $50 \mathrm{ng} / \mu \mathrm{l}$ samples were evaluated by gel electrophoresis. Genotyping was performed on the Affymetrix GeneChip ${ }^{\circledR}$ Human Mapping $500 \mathrm{~K}$ Array Set (Affymetrix Japan, Tokyo, Japan), according to the manufacturer's instructions. To check the quality of each array, the SNPs were initially genotyped by a DM algorithm using Genotyping Console (Affymetrix). All arrays passed a call rate of $93 \%$ at a confidence threshold of 0.33 . For the association analysis, we genotyped the SNPs by the Bayesian robust linear model with a Mahalanobis distance classifier (BRLMM) algorithm using Affymetrix Power Tools ver.1.10.0 (Affymetrix). Genotyping using Taqman ${ }^{\circledR}$ SNP Genotyping Assays (Applied Biosystems, Foster City, CA) on a StepOnePlus ${ }^{\mathrm{TM}}$ real-time PCR system (Applied Biosystems) was performed for the following SNPs in accordance with the manufacturer's instructions: rs800292, rs547154, rs2230199, rs10033900 (Assay ID: C_2530382_10, C_940286_10, C_26330755_10, C_34681305_10).

Statistical analyses

Statistical analyses were carried out with the $\mathrm{R}$ version of 2.7.0 [52]. Deviation of the genotype frequencies in the controls from those expected under Hardy-Weinberg Equilibrium (HWE) was assessed by chi-square test. SNPs with call rate $<90 \%$ for case and control samples, respectively, a Hardy-Weinberg $p<0.0001$ for control samples, a minor allele frequency $<0.05$ for all samples, or those that exhibited poorly defined genotype clusters were disqualified from association analysis. For each case-control study, Fisher's exact test was used to compare allelic or genotypic 
frequencies in three different models (genotype, dominant, and recessive forms) of each case group with controls. In the allelic model, the allelic frequencies were compared between cases and controls using a $2 \times 2$ contingency table. In the genotype model, the frequencies of the three genotypes were compared using a $2 \times 3$ contingency table, in the dominant model, the frequencies of the homozygote for the non-risk allele were compared using a $2 \times 2$ contingency table, and in the recessive model, the frequencies of the homozygote for the risk allele were compared using a $2 \times 2$ contingency table. Also, association analysis was performed with the use of the Cochran-Armitage trend test. After statistical analysis in three different models (genotype, recessive, and dominant model) and trend test, the minimum $\mathrm{p}$ value of each SNPs were obtained from lowest $\mathrm{p}$ value of these tests. The minimum $p$ values were used to test whether they were lower than the significance levels. Odds ratios (ORs) with 95\% confidence intervals (CIs) were estimated for the effects of risk allele and also for both the dominant and recessive forms of the genotypes. To account for multiple statistical testing, the false discovery rate (FDR) was calculated based on the minimum $p$ values from each typical type of AMD and PCV case-control study by the method of Benjamini and Hochberg [53], and it was employed at a significance level of 0.05. Pair wise SNP linkage disequilibrium (LD) values were calculated from the genotype data using Haploview ver.4.0.

\section{Logistic regression analysis and calculation of joint OR}

Logistic regression analysis was performed to assess the joint contributions of the three candidate SNPs (rs800292, rs10490924, and rs2241394) to the risk of typical wettype AMD or PCV using R software. To model the genetic effects, we adopted the following three genetic models with classification variables: the 2-genotype model $(\mathrm{AA}+\mathrm{AB}$ and $\mathrm{BB}$ or $\mathrm{AA}$ and $\mathrm{AB}+\mathrm{BB})$ and the 3-genotype model ( $\mathrm{AA}, \mathrm{AB}$, and $\mathrm{BB}$ ). The logistic regression models were compared by the Akaike information criterion (AIC) to obtain the best-fitting model with the lowest AIC. Univariate logistic regression analysis was initially carried out for three genotype models on each SNPs, and multivariate analysis was then performed. The logistic regression models for all the possible combinations of SNPs were compared by the AIC to obtain the best-fitting model with the lowest AIC. Joint ORs for pairs of loci (rs10490924 and rs800292; rs10490924 and rs2241394; rs10490924 and rs2241394) were calculated for each 2-locus genotype separately, using the non-risk double homozygote genotype as a reference. The estimation of joint ORs was carried out with the R package Epitools. This analysis added 0.5 to each cell in a case including 0 in contingency table [54].

\section{Results}

\section{Genotyping of typical wet-type AMD and PCV}

We conducted genotyping on DNA samples obtained from Japanese patients with typical wet-type AMD and PCV. To increase the power of detecting genetic difference, we selected samples from AMD cases at stage $5 b$, at least in one eye without PCV, for typical wet-type AMD analysis. Stage 5 is defined as exudative AMD, including nondrusenoid pigment epithelial detachments, serious or hemorrhage retinal detachments, choroidal neovascular membrane with subretinal or sub-RPE hemorrhages or fibrosis, or scars consistent with treatment of AMD. AMD with choroidal neovascular membrane or disciform scar is defined as stage $5 \mathrm{~b}$ [51]. Although the controls were age-matched (Table 1), the gender of the population was biased because of the characteristically higher incidence of men in Japanese AMD population [6]. One hundred cases versus 190 controls were genotyped for 500,568 tag-SNPs using the Affymetrix Human Mapping $500 \mathrm{~K}$ Array Set. For typical AMD, statistical analysis was performed for a selected 314,950 tag-SNPs through quality control filters (HWE $p<0.0001$, minor allele frequency $<5 \%$, and call rate $<90 \%$ ) to reduce low-quality signal. These SNPs are shown in Fig. 1a and b, and the 77 SNPs associated with typical wet-type AMD with genotypic $p$ values $<0.0001$ are listed in the Supplementary Table S1. Our study identified several genomic locations as being potentially associated with AMD risk. Among these SNPs, rs10490924, rs3750848, and rs2672587 with false discovery rate $<0.05$ (Benjamini-Hochberg method) were significantly associated with typical wet-type AMD in Japanese patients with a minimum $p$ value of $4.1 \times 10^{-14}, 4.6 \times 10^{-14}$ and $2.2 \times 10^{-9}$, respectively (Table 2 ). For PCV, statistical analysis was performed for 313,772 quality controlled filtered SNPs. These SNPs are shown in Fig. 1c, d, and the 98 SNPs associated with PCV with genotypic $p$ values $<$ 0.0001 are listed in Supplementary Table S2. Two SNPs: rs10490924 and rs3750848 are statistically associated with PCV with a minimum $p$ value of $3.7 \times 10^{-8}$ and $2.4 \times 10^{-8}$, respectively. These results confirm a strong association of the AMRS2/HTRA1 gene region with both typical wet-type AMD and PCV in Japanese, however, we could not comprehensively evaluate the association between Japanese AMD and complement related genes probably due to the low statistical power by this method.

Analysis of the SNPs in ARMS2/HTRA1, CFH, C2/BF, C3, and $C F I$ gene regions

Despite the success of many genome wide association study published, not all the genome wide screening data are analyzed to their full potential. Thus, recently the advantage 



Fig. 1 Genome wide screening for Japanese typical wet-type AMD and PCV. Whole-genome association analyasis of typical wet-type AMD (a) and PCV (c). The scatter plots were drawn for $-\log _{10}$ (minimum $p$ value) against SNP position in the chromosomes. Quantile-quantile plot for association results of typical wet-type

of association analysis on candidate genes selected by certain criteria has been proposed. We extracted genotyping data in gene regions, which were associated with typical wet-type AMD in Caucasian populations, namely ARMS2/ HTRA1, CFH, C2/BF, C3, and CFI. Using TaqMan analysis, we additionally genotyped four SNPs; rs800292 $(C F H)$, rs547154 (C2), rs2230199 (C3), and rs10033900 $(C F I)$, which were significantly associated with Caucasian typical wet-type AMD but were not covered in the Affymetrix Human Mapping 500 K Array. rs800292 shows association with both typical wet-type AMD and PCV $\left(10^{-4}<p<10^{-3}\right.$, Table 2$)$ as reported previously $[39,44]$. No difference in genetic frequency between case and control was detected for rs547154, rs10033900, and rs2230199. After Benjamini-Hochberg correction, 12 and seven SNPs showing significant association with typical wet-type AMD and PCV, respectively (Table 2, Fig. 2), were found at the threshold FDR $<0.05$. For typical wet-type AMD, four SNPs in the $C F H$ gene region including rs800292 appeared to be significantly associated following the ARMS2/HTRA1 gene region. While in $\mathrm{PCV}, \mathrm{rs} 800292$ in the $\mathrm{CFH}$ gene region and rs2241394 in the $C 3$ gene region were
AMD (b) and PCV (d). The distribution of observed $p$ values versus expected $p$ values. Dots represent all the 314950 SNPs and 313772 SNPs analyzed for association studies for typical wet-type AMD and $\mathrm{PCV}$, respectively

significantly associated with the ARMS2/HTRA1 gene region. These results indicate common and distinctive aspects of genetic background between typical wet-type AMD and PCV in Japanese population. We also used logistic regression to further compare the genetic risk properties of typical wet-type AMD and PCV. Table 3 summarizes the best-fit and most parsimonious model analyzed by logistic regression, which was used to explore the joint contributions of the three candidate SNPs (rs800292, rs10490924, and rs2241394) to the risk of typical wet-type AMD and PCV. The AIC for three genetic models of the candidate SNPs were compared, followed by logistic regression analysis to assess the joint contributions of the genetic models with the lowest AIC. These results suggest that the joint effect of rs800292, rs 10490924, and rs2241394 best described the risk for development of PCV. However, an effect of rs2241394 was not observed for typical wet-type AMD. Independent and joint ORs of typical wet-type AMD combining the genotypes from the rs800292 and rs10490924 or PCV for the two variants among rs800292, rs10490924 and rs2241394 were estimated (Table 4). When three SNPs were combined, some values 


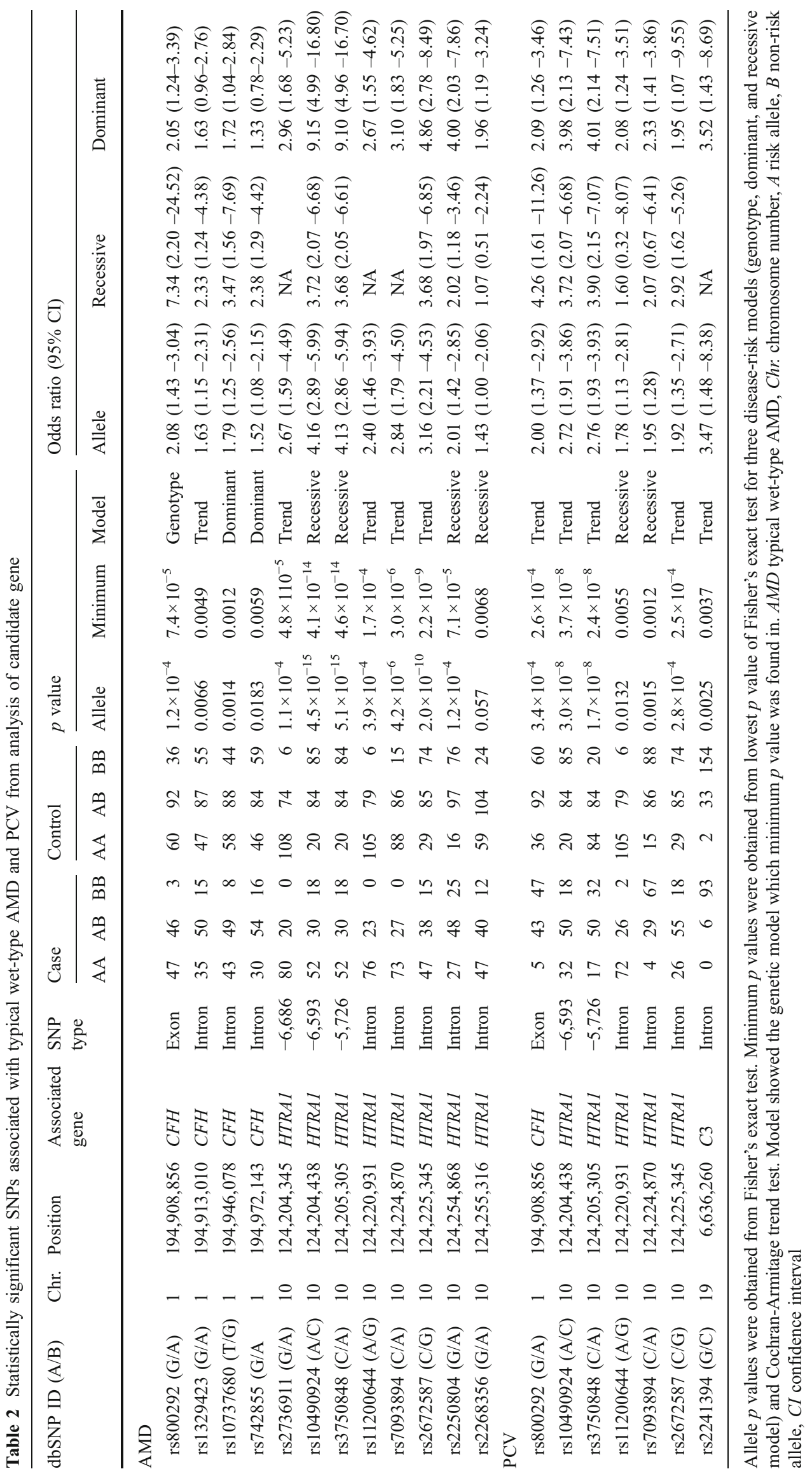



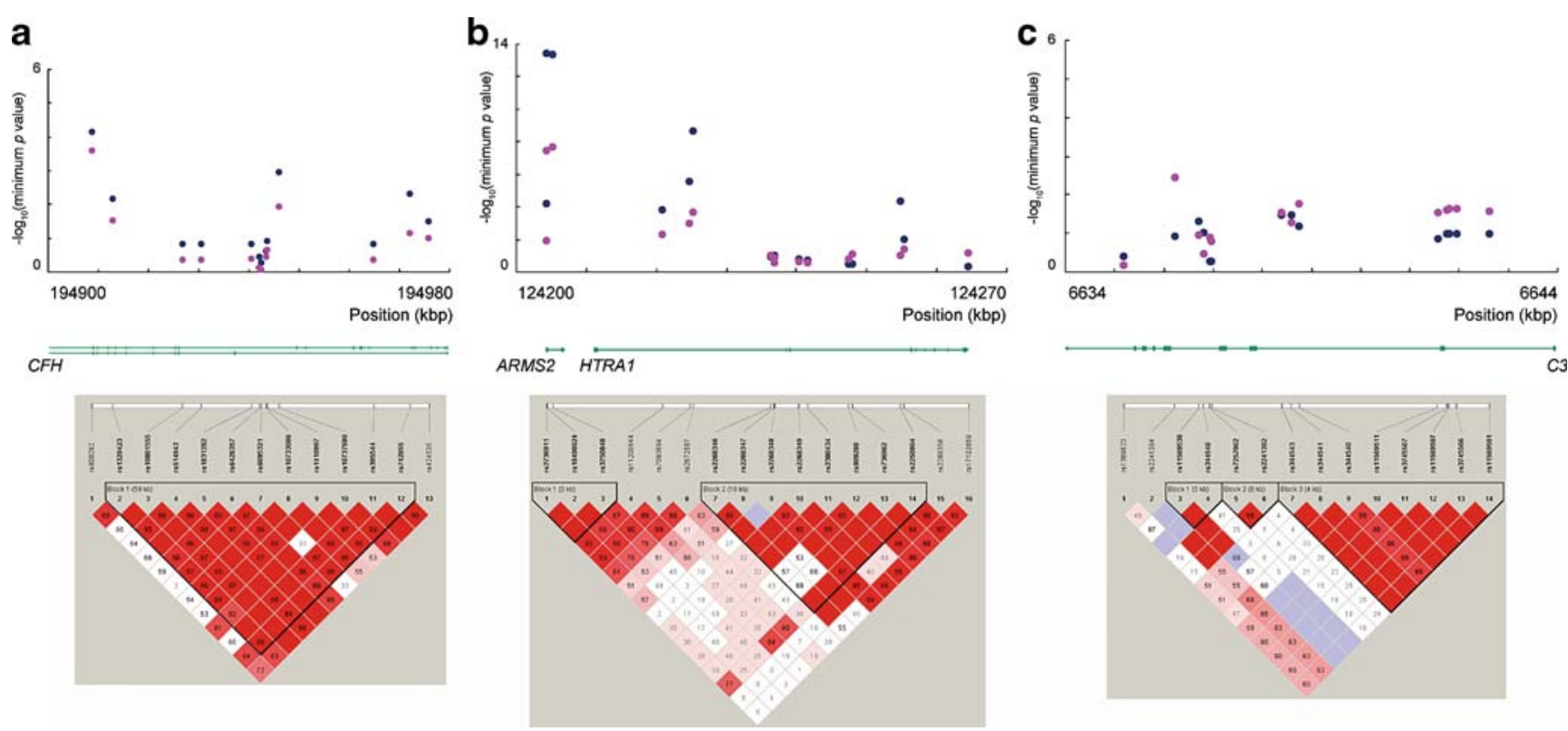

Fig. 2 Schematic view of association and LD results from association analysis for typical wet-type AMD and PCV in the significantly associated region. Upper panel, SNPs association results in the chromosome 1 (a), chromosome 10 (b), and chromosome 19 (c). This panel shows $p$ values for association testing drawn from the candidate genes focused study including results using Taqman

appeared to be unreliable because of small numbers of individuals in some of the genotype combinations. Therefore, two SNPs were analyzed. The heterozygous (CA) risk genotype of rs10490924 was detected more often in PCV cases than in controls with $p$ value of 0.0008 and OR of 2.8 genotyping assay. Known genes in the area are shown. Down panel, LD structure of each region. Pairwise LD was calculated from all the data set in this study using the methods of Gabriel as implemented in Haploview. In each box are shown are the LD relationships between each SNP pair using Haploview's standard color scheme. Blue circles, typical wet-type AMD; Red circles, PCV

(95\% CI $=1.5-5.2)$, however the difference in the frequency of the heterozygous (CA) at the same loci between typical wet-type AMD cases and controls did not reach statistical significance. Both typical wet-type AMD and PCV cases carried the rs800292 heterozygous (AG) and homozygous

Table 3 Calculation of AIC by logistic regression analysis in three genotype models

\begin{tabular}{|c|c|c|c|c|c|}
\hline \multirow[t]{2}{*}{ dbSNP ID } & \multirow[t]{2}{*}{ Model } & \multicolumn{2}{|l|}{ AMD } & \multicolumn{2}{|l|}{ PCV } \\
\hline & & AIC & AIC difference & $\mathrm{AIC}$ & AIC difference \\
\hline \multicolumn{6}{|l|}{ Single } \\
\hline \multirow[t]{3}{*}{ rs800292 } & Gen & 349.6 & & 352.1 & \\
\hline & $\operatorname{Rec}$ & 350.4 & & 353.8 & \\
\hline & Dom & 359.6 & & 357 & \\
\hline \multirow[t]{3}{*}{ rs 10490924} & Gen & 317.9 & & 347.8 & \\
\hline & Rec & 354.7 & & 354.7 & \\
\hline & Dom & 318.4 & & 357.4 & \\
\hline \multirow[t]{3}{*}{ rs2241394 } & Gen & 375.4 & & 366.7 & \\
\hline & Rec & 345.1 & & 373 & \\
\hline & Dom & 374.7 & & 365.3 & \\
\hline \multicolumn{6}{|l|}{ Joint } \\
\hline rs800292gen + rs10490924gen & & 291.8 & 0 & 325.9 & 5.5 \\
\hline rs800292gen + rs2241394dom & & 349.2 & 57.4 & 344 & 23.6 \\
\hline rs10490924gen + rs2241394dom & & 319.2 & 27.4 & 344 & 23.6 \\
\hline rs800292gen + rs10490924gen + rs2241394dom & & 293.4 & 1.6 & 320.4 & 0 \\
\hline
\end{tabular}

$A M D$ typical wet-type AMD, Gen genotype model, Rec recessive model, Dom dominant model, AIC Akaike information criterion (the AIC difference is the difference from the AIC of the best fitting model) 
Table 4 Two-locus odds ratios and $p$ values for different genotypic combinations of the rs800292, rs10490924, and rs2241394 polymorphisms

\begin{tabular}{|c|c|c|c|c|c|c|c|c|}
\hline \multicolumn{5}{|c|}{ Genotype combination } & \multicolumn{4}{|l|}{ Number $(\%)$} \\
\hline & & & & & Control & Case & Odds ratio $(95 \% \mathrm{CI})$ & $p$ value \\
\hline \multirow[t]{17}{*}{ AMD } & Single & & & & & & & \\
\hline & rs800292 & AA & & & $36(0.19)$ & $3(0.03)$ & 1 & \\
\hline & & AG & & & $92(0.49)$ & $46(0.48)$ & $6.00(1.75-20.52)$ & 0.0011 \\
\hline & & GG & & & $60(0.32)$ & $47(0.49)$ & $9.40(2.73-32.42)$ & $2.3 \times 10^{-5}$ \\
\hline & rs10490924 & $\mathrm{CC}$ & & & $85(0.45)$ & $18(0.18)$ & 1 & \\
\hline & & $\mathrm{CA}$ & & & $84(0.44)$ & $30(0.3)$ & $1.69(0.87-3.26)$ & 0.1411 \\
\hline & & AA & & & $20(0.11)$ & $52(0.52)$ & $12.28(5.95-25.33)$ & $2.9 \times 10^{-13}$ \\
\hline & Joint & & & & & & & \\
\hline & rs10490924 & $\mathrm{CC}$ & rs800292 & AA & $15(0.05)$ & $1(0)$ & 1 & \\
\hline & & & & $\mathrm{AG}$ & $38(0.13)$ & $11(0.04)$ & $3.09(0.51-18.69)$ & 0.3234 \\
\hline & & & & GG & $32(0.11)$ & $5(0.02)$ & $1.75(0.26-11.74)$ & 1 \\
\hline & & $\mathrm{CA}$ & & AA & $15(0.05)$ & $0(0)$ & $0.33(0.01-8.83)$ & 0.4866 \\
\hline & & & & AG & $45(0.16)$ & $15(0.05)$ & $3.52(0.6-20.71)$ & 0.3353 \\
\hline & & & & GG & $23(0.08)$ & $14(0.05)$ & $6.38(1.05-38.54)$ & 0.061 \\
\hline & & AA & & AA & $6(0.02)$ & $2(0.01)$ & $3.97(0.43-36.66)$ & 0.5633 \\
\hline & & & & $\mathrm{AG}$ & $8(0.03)$ & $20(0.07)$ & $24.92(3.89-159.6)$ & $7.1 \times 10^{-5}$ \\
\hline & & & & GG & $5(0.02)$ & $28(0.1)$ & $53.55(7.94-361.02)$ & \\
\hline \multirow[t]{25}{*}{ PCV } & Single & & & & & & & \\
\hline & rs800292 & GG & & & $36(0.19)$ & $5(0.05)$ & 1 & \\
\hline & & GA & & & $92(0.49)$ & $43(0.45)$ & $3.37(1.23-9.18)$ & 0.0156 \\
\hline & & AA & & & $60(0.32)$ & $47(0.49)$ & $5.64(2.05-15.49)$ & \\
\hline & rs10490924 & $\mathrm{CC}$ & & & $85(0.45)$ & $18(0.18)$ & 1 & \\
\hline & & $\mathrm{CA}$ & & & $84(0.44)$ & $50(0.5)$ & $2.81(1.52-5.21)$ & 0.0008 \\
\hline & & AA & & & $20(0.11)$ & $32(0.32)$ & $7.56(3.55-16.08)$ & $6.0 \times 10^{-8}$ \\
\hline & rs2241394 & $\mathrm{CC}+\mathrm{CG}$ & & & $35(0.19)$ & $6(0.06)$ & 1 & \\
\hline & & GG & & & $154(0.81)$ & $93(0.94)$ & $3.52(1.43-8.69)$ & 0.0041 \\
\hline & Joint & & & & & & & \\
\hline & rs10490924 & $\mathrm{CC}$ & rs80092 & AA & $15(0.05)$ & $0(0)$ & 1 & \\
\hline & & & & $\mathrm{AG}$ & $38(0.13)$ & $7(0.02)$ & $6.04(0.32-112.28)$ & 0.0994 \\
\hline & & & & GG & $32(0.11)$ & $11(0.04)$ & $10.97(0.61-198.43)$ & 0.0253 \\
\hline & & CA & & AA & $15(0.05)$ & $4(0.01)$ & $9.00(0.45-181.74)$ & 0.1131 \\
\hline & & & & $\mathrm{AG}$ & $45(0.16)$ & $24(0.09)$ & $16.69(0.96-291.08)$ & 0.0043 \\
\hline & & & & GG & $23(0.08)$ & $21(0.07)$ & $28.36(1.6-503.36)$ & 0.0005 \\
\hline & & AA & & AA & $6(0.02)$ & $1(0)$ & $7.15(0.26-199.68)$ & 0.1014 \\
\hline & & & & $\mathrm{AG}$ & $8(0.03)$ & $12(0.04)$ & $45.59(2.39-869.16)$ & 0.0002 \\
\hline & & & & GG & $5(0.05)$ & $15(0.05)$ & $87.36(4.44-1,719.03)$ & $4.6 \times 10^{-6}$ \\
\hline & rs10490924 & $\mathrm{CC}$ & rs2241394 & $\mathrm{CC}+\mathrm{CG}$ & $15(0.08)$ & $2(0.02)$ & 1 & \\
\hline & & & & GG & $70(0.37)$ & $16(0.16)$ & $1.71(0.36-8.26)$ & 0.7302 \\
\hline & & $\mathrm{CA}$ & & $\mathrm{CC}+\mathrm{CG}$ & $17(0.09)$ & $2(0.02)$ & $0.88(0.11-7.06)$ & 1 \\
\hline & & & & GG & $67(0.35)$ & $48(0.48)$ & $5.37(1.17-24.6)$ & 0.0172 \\
\hline & & AA & & $\mathrm{CC}+\mathrm{CG}$ & $3(0.02)$ & $2(0.02)$ & $5.00(0.49-50.83)$ & 0.2098 \\
\hline & & & & GG & $17(0.09)$ & 29. (0.29) & $12.79(2.6-62.88)$ & 0.0004 \\
\hline
\end{tabular}

AMD, typical wet-type AMD; 95\%CI, 95\% confidence intervals; $p$ values were obtained from Fisher's exact text 
(GG) risk genotype more often than the non-risk genotype (AA) (typical wet-type AMD cases: $p=0.001, \mathrm{OR}=6.0$; AG and $p=2.2 \times 10^{-5}$, $\mathrm{OR}=9.4$; GG, PCV cases: $p=0.016$, $\mathrm{OR}=$ 3.4; $\mathrm{AG}$ and $p=0.0002, \mathrm{OR}=5.6$; GG). A joint $\mathrm{OR}$ of 53.5 for typical wet-type AMD in individuals with homozygous (AA and GG) risk alleles at both loci was observed when compared with the non-risk genotype (CC and AA), with a wide range of calculated $95 \%$ CI from 7.9 to 361.0. Similarly, a joint OR of 87.4 for PCV in individuals with homozygous (AA and GG) risk alleles at the same loci was observed. A joint analysis of ORs for the rs10490924 and rs2241394 showed that the risk of PCV was 5.4-fold $(p=0.017)$ and 12.8-fold $(p=0.0004)$ if individuals had homozygous (GG) risk genotype of rs2241394 and heterozygous (CA) and homozygous (AA) risk genotype of rs10490924, respectively, compared with the non-risk genotype. A plot of the two-locus genotype specific for typical wet-type AMD and PCV risks further illustrates the stronger impact of rs10490924 on these diseases (Fig. 3).

\section{LD block analyses}

rs800292 and rs1061170 are located within exon 2 and exon 9, respectively, of the $C F H$ gene on chromosome 1. rs800292 was included in LD block 1 indicated in Fig. 2a. rs1061170 was located in a different LD block from rs800292 (data not shown). The leading two SNPs rs10490924 and rs3750848, which have been reported to associate with AMD [30], maps to a small LD block 1 on chromosome 10 (Fig. 2b). rs10490924 and rs3750848 were located within exon 1 and intron 1 of $A R M S 2$. Another SNP, rs2672587, included in the intronic region of HTRA1 is located downstream of the leading two SNPs between block 1 and 2 (Fig. 2b). rs2230199 and rs2241394 were located within exon 3 and intron 13 of the $C 3$ gene, respectively on chromosome 19. rs2241394 was not included in LD blocks indicated in Fig. 2c.

\section{Discussion}

We performed a first genome wide genotyping of Japanese patients with typical wet-type AMD and PCV using Affymetrix GeneChip ${ }^{\circledR}$ Human Mapping $500 \mathrm{~K}$ Array and TaqMan assay. Previous genome wide scans on individuals with the dry and wet-type AMD in Caucasian populations indicated that $C F H$ and ARMS2/HTRA1 are the two most significantly associated gene regions for AMD

\section{a. rs 10490924}

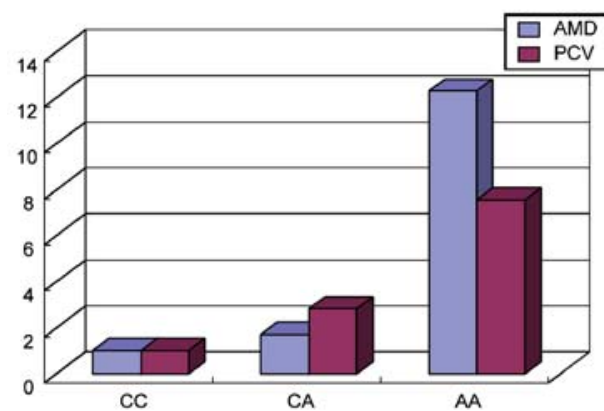

b. $\mathrm{rs} 800292$

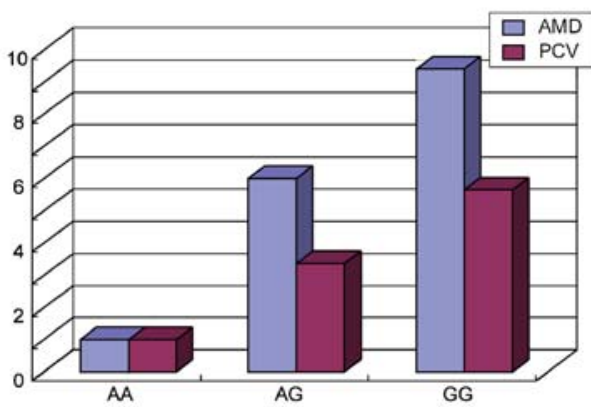

C. $A M D, r s 10490924$ and $r s 800292$

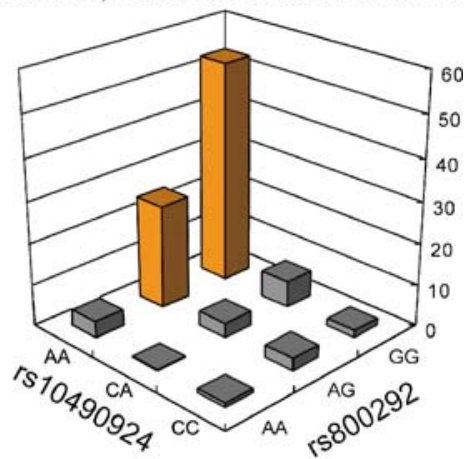

d. PCV, rs10490924 and rs800292

e.PCV, rs10490924 and rs2241394

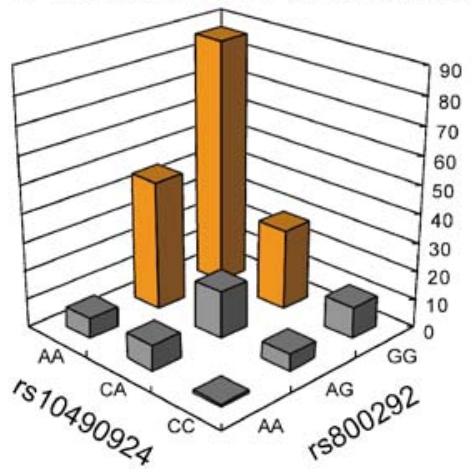

Fig. 3 Schematic view of two-locus odds ratios. Upper panel, independently genetic effect of rs10490924 (a) and rs800292 (b). Blue boxes, typical wet-type AMD; Purple boxes, PCV. Down panel, joint effect of indicated SNPs to typical wet-type AMD and PCV. Joint odds

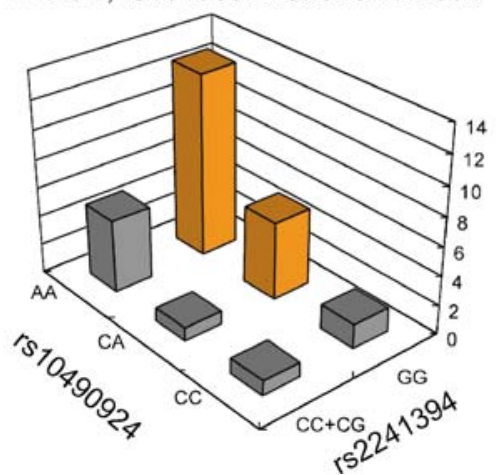

ratios for the combination of rs10490924 and rs800292 for typical wettype AMD (c), rs10490924 and rs800292 for PCV (d), and rs10490924 and rs2241394 (e) are shown. The combinations had statistically significant odds ratio are colored. AMD typical wet-type AMD 
followed by $C 2 / B F, C 3$, and $C F I[15-18,26]$ (Swaroop A, et al. IOVS 2009; 50: ARVO E-Abstract 1614). Interestingly, Swaroop et al., reported that the most significantly associated gene locus was $C F H$ at $p$ values $<10^{-74}$ followed by $A R M S 2$ at $p$ values $<10^{-57}$. In contrast, the Japanese typical wet-type AMD patients showed stronger association with AMRS2/HTRA1 compared to $C F H$ (Table 2). Affymetrix GeneChip ${ }^{\circledR}$ Human Mapping $500 \mathrm{~K}$ Array used in this study contained 19 SNPs covering the CFH gene region (13 SNPs passed the quality control filters). The LD blocks in this region are notably large and with the additional genotyping of rs 800292 by TaqMan assay, the whole $\mathrm{CFH}$ gene region has been covered. Thus, the association of rs800292 with typical wet-type AMD and PCV has been confirmed, and the results in this study clearly indicate a lower contribution of $C F H$ compared to AMRS2/HTRA1 on typical wet-type AMD and PCV in the Japanese AMD population. This is consistent with our earlier findings together with others, which have shown lower association of $C F H$ compared to ARMS2/HTRA1 for AMD in Japanese individuals [33, 38-41]. Furthermore, recent studies demonstrated that $10 \mathrm{q} 26$ is more strongly associated than $1 \mathrm{q} 32$ in wet-type AMD in Caucasian populations $[27,31,32]$. Combining the results of our study with previous studies suggests that $C F H$ and ARMS2/ HTRA1 independently influence the progression of both dry-type and wet-type AMD. Taking into consideration, the lower association of rs 1061170 and other variations in $\mathrm{CFH}$ gene reported in Chinese [42, 55, 56] and Korean populations [57], our results may have revealed some of the common features of AMD patients among East Asian. However, it should be noted that despite the low frequency of the risk allele, a significant association between the rs1061170 variant and typical wet-type AMD have been demonstrated in some Chinese population $[58,59]$. As Chu et al. have suggested in their paper, the existence of ethnic group variation within the East Asian population is possible [59]. We also performed additional genotyping of SNPs in the $C 2 / B F, C 3$, and $C F I$ gene regions, with SNPs not included on the SNP array. rs547154 (C2) is one of the protective haplotype-tagging SNPs originally reported by Gold et al. [45] who discovered a risk haplotype and two protective haplotypes. Kondo et al. have examined the effects of 11 SNPs in the above gene region in Japanese $\mathrm{PCV}$ cases [50]. Although individual SNPs were not associated, statistically significant association of a protective haplotype was found. This protective haplotype was different from the two haplotypes described by Gold et al. It is unclear whether this difference is due to the difference in pathogenesis between dry-type AMD and PCV or ethnicity. As for rs2230199 (C3), the risk allele frequency was 0 in subjects analyzed in this study, which was consistent with the data from the Hap Map project. The risk allele for rs2230199 was 0.175 in European population (Hap Map project). In our study, rs2241394 in intron 13 of $C 3$ gene showed significant association with $\mathrm{PCV}$ but not with typical wet-type AMD (Table 2).

The risk of typical wet-type AMD associated with rs10490924 was very high $(\mathrm{OR}=12.3 ; 95 \% \mathrm{CI}=6.0-25.3)$, whereas the risk of PCV conferred by rs $10490924(\mathrm{OR}=$ 7.6; $95 \% \mathrm{CI}=3.5-16.1)$ was similar to the risk of rs 800292 $(\mathrm{OR}=5.6 ; 95 \% \mathrm{CI}=2.1-15.5)$ (Table 4). Tam et al. reported that combined rs 11200638 and rs 800292 caused a 23.3 -fold increased risk for exudative AMD [60]. rs 11200638 is a SNP shown to be in LD with rs10490924 [29-31]. Similarly, our results showed that the joint risk of rs10490924 and rs800292 for PCV was 87.4-fold (Table 4, Fig. 3).

Structures of $\mathrm{CFH}$ and $\mathrm{C} 3$ have been reported previously $[61,62]$. CFH consists of twenty short consensus repeats, each presumed to fold into a distinct structure termed the complement control protein module (CCP) [61]. Y402H (rs1061170) and I62V (rs800292) are located in the CCP1 and $\mathrm{CCP} 7$, which are implicated in $\mathrm{C} 3 / \mathrm{C} 3 \mathrm{~b}$ binding and polyanion binding, respectively [61]. Accordingly, rs1061170 and rs800292 polymorphisms may differentially influence the $\mathrm{CFH}$ activity. $\mathrm{C} 3$ consist of multiple domains [62]. R80G (rs2230199) is included in the macroglobulin (MG) 1 domain which is located in the electropositive patch essential for binding target surfaces such as bacterial cells or apoptotic host cells [62]. rs2241394, which is located in intron 13 of the $C 3$ gene, showed strong associated with PCV in this study (Fig. 2c). Interestingly, exons 13 and 14 adjacent to rs2241394 encode the MG5 domain, which is also located within the electropositive patch together with MG1 domain. To investigate the molecular mechanism of PCV pathogenesis related to rs2241394, further exploration of SNPs within this region is needed.

The LD block including ARMS2 contains two of the significantly associated SNPs, rs 10490924 and rs3750848 (Fig. 2b, Table 2). Another significantly associated SNP, rs2672587, was in the intronic region of HTRA1, located approximately $6 \mathrm{Kbp}$ downstream of ARMS2 (Table 2, Fig. 2b). The rs11200638 located 512 bp upstream of the transcription initiation point is absent in the Affymetrix Human Mapping $500 \mathrm{~K}$ Array but has been reported to associate with AMD at a level comparable to rs10490924 $[31,34,37]$. Our genotyping of rs11200638 by sequencing also confirmed this result [33]. Our data indicate that both rs11200638 and rs10490924 share the same LD block which contains ARMS2 and HTRA1 but different from rs2672587 (data not shown). This result is consistent with previous Caucasian studies [29-31]. Some of the candidate loci appeared in this study may be specific to the Japanese AMD population or critical for the development of AMD in combination with ARMS2 (rs10490924) and/or HTRA1 
(rs11200638), and other behavioral, nutritional, and environmental factors. Further replication and detailed experiments are needed.

Acknowledgments We are grateful to all patients and their families who participated in this study. This study was supported in part by the grant to TI from the National Hospital Organization and the Japanese Ministry of Health, Welfare, and Labour of Japan.

Open Access This article is distributed under the terms of the Creative Commons Attribution Noncommercial License which permits any noncommercial use, distribution, and reproduction in any medium, provided the original author(s) and source are credited.

\section{References}

1. Ding X, Patel M, Chan CC. Molecular pathology of age-related macular degeneration. Prog Retin Eye Res. 2009;28:1-18.

2. Kawasaki R, Wang JJ, Ji GJ, et al. Prevalence and risk factors for age-related macular degeneration in an adult Japanese population: Funagata study. Ophthalmology. 2008;115:1376-81.

3. Lim JI. Age-related macular degeneration. New York: Marcel Dekker; 2002

4. Bonilha VL. Age and disease-related structural changes in the retinal pigment epithelium. Clin Ophthalmol. 2008;2:413-24.

5. Maruko I, Iida T, Saito M, et al. Clinical characteristics of exudative age-related macular degeneration in Japanese patients. Am J Ophthalmol. 2007;144:15-22.

6. Gomi F, Tano Y. Popypoidal choroidal vasculopathy and treatments. Curr Opin Ophthalmol. 2008;19:208-12.

7. Cacket P, Wong D, Yeo I. A classification system for polypoidal choroidal vasculopathy. Retina. 2009;29:187-91.

8. Byeon SH, Lee SC, Oh HS, et al. Incidence and clinical patterns of polypoidal choroidal vasculopathy in Korean patients. Jpn J Ophthalmol. 2008;52:57-62.

9. Meyers SM. A twin study on age-related macular degeneration. Trans Am Ophthalmol Soc. 1994;92:775-843.

10. Hammond CJ, Webster AR, Snieder H, et al. Genetic influence on early age-related maculopathy: a twin study. Ophthalmology. 2002;109:730-6.

11. Seddon JM, Cote J, Page WF, et al. The US twin study of age-related macular degeneration: relative roles of genetic and environmental influences. Arch Ophthalmol. 2005;123:321-7.

12. Heiba IM, Elston RC, Klein BE, et al. Sibling correlations and segregation analysis of age-related maculopathy: the Beaver Dam Eye Study. Genet Epidemiol. 1994;11:51-67.

13. Seddon JM, Ajani UA, Mitchell BD. Familial aggregation of age-related maculopathy. Am J Ophthalmol. 1997;123:199206.

14. Klaver CC, Wolfs RC, Assink JJ, et al. Genetic risk of age-related maculopathy. Population-based familial aggregation study. Arch Ophthalmol. 1998;116:1646-51.

15. Abecasis GR, Yashar BM, Zhao Y, et al. Age-related macular degeneration: a high-resolution genome scan for susceptibility loci in a population enriched for late-stage disease. Am J Hum Genet. 2004;41:482-94.

16. Iyengar SK, Song D, Klein BE, et al. Dissection of genomewidescan in extended families reveals a major locus and oligogenic susceptibility for age-related macular degeneration. Am J Hum Genet. 2004;74:20-39.

17. Majewski J, Schultz DW, Weleber RG, et al. Age-related macular degeneration - a genome scan in extended families. Am J Hum Gentet. 2003;73:540-50.
18. Seddon JM, Santangelo SL, Book K, et al. A Genomewide scan for age-related macular degeneration provides evidence for linkage to several chromosomal regions. Am J Hum Genet. 2003;73:780-90.

19. Klein ML, Schultz DW, Edwards A, et al. Age-related macular degeneration. Clinical features in a large family and linkage to chromosome 1q. Arch Ophthalmol. 1998;116:1082-8.

20. Schick JH, Iyengar SK, Klein BE, et al. A whole-genome screen of a quantitative trait of age-related maculopathy in sibships from the Beaver Dam Eye Study. Am J Hum Genet. 2003;72:1412-24.

21. Kenealy SJ, Schmidt S, Agarwal A, et al. Linkage analysis for age-related macular degeneration supports a gene on chromosome 10q26. Mol Vis. 2004;10:57-61.

22. Schmidt S, Scott WK, Postel EA, et al. Ordered subset linkage analysis supports a susceptibility locus for age-related macular degeneration on chromosome 16p12. BMC Genet. 2004;5:18.

23. Weeks DE, Conely YP, Tsai HJ, et al. Age-related maculopathy: a genomewide scan with continued evidence of susceptibility loci within the 1q31, 10q26, and 17q25 regions. Am J Hum Genet. 2004;75:174-89.

24. Barral S, Francis PJ, Schultz DW, et al. Expanded genome scan in extended families with age-related macular degeneration. Invest Ophthalmol Vis Sci. 2006;47:5453-9.

25. Fisher SA, Abecasis GR, Yashar BM, et al. Meta-analysis of genome scans of age-related macular degeneration. Hum Mol Genet. 2005; 14:2257-64.

26. Edwards AO, Fridley BL, James KM, et al. Evaluation of clustering and genotype distribution for replication in genome wide association studies: the age-related eye disease study. PLoS ONE. 2008;3:e3813.

27. Zhang H, Morrison MA, DeWan A, et al. The NEI/NCBI dbGAP database: genotypes and haplotypes that may specifically predispose to risk of neovascular age-related macular degeneration. BMC Med Genet. 2008;9:51.

28. Klein RJ, Zeiss $\mathrm{C}$, Chew EY, et al. Complement factor $\mathrm{H}$ polymorphism in age-related macular degeneration. Science. 2005;308:385-9.

29. Rivera A, Fisher SA, Fritsche LG, et al. Hypothetical ARMS2 is a second major susceptibility gene for age-related macular degeneration, contributing independently of complement factor $\mathrm{H}$ to disease risk. Hum Mol Genet. 2005;14:3227-36.

30. Jakobsdottir J, Conley YP, Weeks DE, et al. Susceptibility genes for age-related maculopathy on chromosome 10q26. Am J Hum Genet. 2005;77:389-407.

31. Dewan A, Liu M, Hartman S, et al. HTRA1 promoter polymorphism in wet age-related macular degeneration. Science. 2006;314:989-92.

32. Yang Z, Camp NJ, Sun H, et al. A variant of the HTRA1 gene increases susceptibility to age-related macular degeneration. Science. 2006;314:992-3.

33. Yoshida T, De Wan A, Zhang H, et al. HTRA1 promoter polymorphism predisposes Japanese to age-related macular degeneration. Mol Vis. 2007;13:545-8.

34. Cameron DJ, Yang Z, Chen $H$, et al. HTRA1 variant confers similar risks to geographic atrophy and neovascular age-related macular degeneration. Cell Cycle. 2007;6:1122-5.

35. Kondo N, Honda S, Ishibashi K, et al. ARMS2/HTRA1 variants in polypoidal choroidal vasculopathy and age-related macular degeneration in a Japanese population. Am J Ophthalmol. 2007;144:608-12.

36. Fuse N, Miyazawa A, Mengkegale $M$, et al. Polymorphisms in Complement Factor $\mathrm{H}$ and Heminectin-1 genes in a Japanese Population with dry-type age-related macular degeneration. Am J Ophthalmol. 2006;142:1074-6.

37. Okamoto $\mathrm{H}$, Umeda $\mathrm{S}$, Obazawa $\mathrm{M}$, et al. Complement factor $\mathrm{H}$ polymorphisms in Japanese population with age-related macular degeneration. Mol Vis. 2006;12:156-8. 
38. Gotoh N, Yamada R, Hiratani H, et al. No association between complement factor $\mathrm{H}$ gene polymorphism and exudative age-related macular degeneration in Japanese. Hum Genet. 2006;120:139-43.

39. Mori K, Gehlbach PL, Kabasawa I, et al. Coding and noncoding variants in the $\mathrm{CFH}$ gene and cigarette smoking influence the risk of age-related macular degeneration in a Japanese population. Invest Ophthalmol Vis Sci. 2007;48:5315-9.

40. Uka J, Tamura H, Kobayashi T, et al. No association of complement factor $\mathrm{H}$ gene polymorphism and age-related macular degeneration in the Japanese population. Retina. 2006;26:985-7.

41. Grassi MA, Fingert JH, Scheetz TE, et al. Ethnic variation in AMD-associated complement factor $\mathrm{H}$ polymorphism p. Tyr402His. Hum Mutat. 2006;27:921-5.

42. Chen LJ, Liu DT, Tam PO, et al. Association of complement factor $\mathrm{H}$ polymorphism with exudative age-related macular degeneration. Mol Vis. 2006;12:1536-42.

43. Lee KY, Vitbana EN, Matbur R, et al. Association analysis of $\mathrm{CFH}, \mathrm{C} 2, \mathrm{BF}$, and HTRA1 gene polymorphisms in Chinese patients with polypoidal choroidal vasculopathy. Invest Ophthalmol Vis Sci. 2008;49:2613-9.

44. Kondo N, Honda S, Kuno S, et al. Coding variant I62V in the complement factor $\mathrm{H}$ gene is strongly associated with polypoidal choroidal vasculopahy. Ophthalmology. 2009;116:304-10.

45. Gold B, Merriam JE, Zernant J, et al. Variation in factor B (BF) and complement component $2(\mathrm{C} 2)$ genes is associated with agerelated macular degeneration. Nature Genet. 2006;38:458-62.

46. Richardson AJ, Islam FMA, Guymer RH, et al. Analysis of rare variants in the complement component $2(\mathrm{C} 2)$ and factor $\mathrm{B}(\mathrm{BF})$ genes refine association for age-related macular degeneration. Invest Ophthalmol Vis Sci. 2009;50:540-3.

47. Maller JB, Fagerness JA, Reynolds RC, et al. Variation in complement factor 3 is associated with risk of age-related macular degeneration. Nature Genet. 2007;39:1200-1.

48. Yates JRW, Sepp T, Matharu BK, et al. Complement C3 variant and the risk of age-related macular degeneration. $\mathrm{N}$ Eng $\mathrm{J}$ Med. 2009;357:553-61.

49. Fagerness JA, Maller JB, Neale BM, et al. Variation near complement factor I is associated with risk of advanced AMD. Eur J Hum Genet. 2009;17:100-4.

50. Kondo N, Honda S, Kuno S, et al. Role of RDBP and SKIV2L variants in the major histocompatibility complex class III region in polypoidal choroidal vasculopathy etiology. Ophthalmology. 2009;116:1502-9.

51. Seddon JM, Sharma S, Adelman RA. Evaluation of the clinical agerelated maculopathy staging system. Ophthalmol. 2006;113:260-6.

52. R Development Core Team. R: A language and environment for statistical computing. Vienna: R Foundation for Statistical Computing; 2009. ISBN 3-900051-07-0, URL http://www. R-project.org.

53. Benjamini Y, Hochberg Y. Controlling the false discovery rate: a practical and powerful approach to multiple testing. J R Stat Soc. 1995;B57:289-300.

54. Agresti A. Categorical Data Analysis. New Jersey: John Wiley \& Sons; 2002.

55. Xu Y, Guan N, Xu J, et al. Association of CFH, ARMS2, and HTRA1 polymorphisms with exudative age-related macular degeneration in a northern Chinese population. Mol Vis. 2008;14:1373-81.

56. Ng TK, Chen LJ, Liu DT, et al. Multiple gene polymorphisms in the complement factor $\mathrm{h}$ gene are associated with exudative agerelated macular degeneration in Chinese. Invest Ophthalmol Vis Sci. 2008;49:3312-7.

57. Kim NR, Kang JH, Kwon OW, et al. Association between complement factor $\mathrm{H}$ gene polymorphism and neovascular agerelated macular degeneration in Koreans. Invest Ophthalmol Vis Sci. 2008;49:2071-6.

58. Lau LI, Chen SJ, Cheng CY, et al. Association of the $\mathrm{Y} 402 \mathrm{H}$ polymorphism in complement factor $\mathrm{H}$ gene and neovascular agerelated macular degeneration in Chinese patients. Invest Ophthalmol Vis Sci. 2006;47:3242-6.

59. Chu J, Zhou CC, Lu N, et al. Genetic variants in three genes and smoking show strong associations with susceptibility to exudative age-related macular degeneration in a Chinese population. Chin Med J. 2008;121:2525-33.

60. Tam PO, Ng TK, Liu DT, et al. HTRA1 variants in exudative agerelated macular degeneration and interactions with smoking and CFH. Invest Ophthalmol Vis Sci. 2008;49:2357-65.

61. Scmidt CQ, Herbert AP, Hocking HG, et al. Translational minireview series on complement factor $\mathrm{H}$ : structural and functional correlations for factor H. Cln Exp Immun. 2008;151:14-24.

62. Janssen BJC, Christodoulidou A, McCarthy A, et al. Structures of $\mathrm{C} 3 \mathrm{~b}$ reveals conformational changes that underlie complement activity. Nature. 2006;444:213-6. 